\title{
The frailty flying squad: implementing a front door service
}

\author{
Authors: Aishwarya Desai, Donna Thomas, Katharine Abbott, Olivia Maskell, Katie Rainey and Jarrod Richards
}

\section{Introduction}

Frailty is an important geriatric syndrome characterised by ageing-associated declines in reserve and function across multiple physiologic systems. ${ }^{1}$ Appropriate management of frailty within secondary care is crucial, particularly in the context of acute hospital admissions. ${ }^{2}$ This was a pilot study within Southmead Hospital implementing a frailty 'front door' team based within the emergency department (ED) and acute medical unit (AMU).

\section{Methods}

This pilot ran over 8 weeks and consisted of a team with a consultant geriatrician, advanced nurse practitioners (ANPs) and therapy support. Patients were selected from the medical referral list and direct review of ED patients which were deemed appropriate by the consultant geriatrician or ANPs. A multifaceted clinical review was carried out including falls assessment, cognitive assessment and selected advanced care planning.

\section{Results and discussion}

A total of 42 patients were seen over the 8-week period with an average Rockwood Clinical Frailty Score of 6 . The majority of patients were seen in ED (62\%) followed by AMU (33\%) and direct GP referrals (5\%).

The same day discharge rates were $66 \%$ for the patients reviewed. Of note $93 \%$ of these patients had already been referred to medicine for acute admission. The readmission rate in the group was $14 \%$. The most common presenting complaint was 'fall' (71\%). All patients underwent cognitive assessment and there was an average of 0.55 medications stopped per patient.

The average length of stay (LOS) was 4.4 days for the patients who were admitted. There were three patients who had complex needs and subsequently an increased LOS of 8,8 and 12 days. Excluding these patients, the average LOS was 2.4 days compared with the pre-existing LOS on the short stay care of elderly ward which was 4.8 days. The potential cost savings with this service remain to be accurately calculated but are significant.

\section{Conclusions}

The introduction of this pilot has reduced admission rates of frail elderly patients and LOS. It remains ongoing and we intend to increase resources (ANPs, therapists and consultant) to help meet demands of admissions. We have a wide range of patient stories with successes in family discussion and primary care liaison. Other successful services have used a dedicated 'silver phone' for patients to provide post discharge advice if required. ${ }^{2}$ The overall aim is to create a dedicated area to review identified patients from ED, AMU and GP referrals with closer operation with ambulance services.

\section{Conflict of interest statement}

None declared.

\section{References}

1 Clegg A, Young J, Iliffe S, Rikkert MO, Rockwood K. Frailty in elderly people. Lancet 2013;381:752-62.

2 Network Acute Frailty. Managing acute frailty. NHS Elect, 2016. www.acutemedicine.org.uk/wp-content/uploads/2016/05/4.1Managing-Acute-Frailty.pdf [Accessed 26 March 2019]. 